\title{
Loop colostomies are safe in anorectal malformations
}

\author{
Dhanya Mullassery ${ }^{\$ 1}$, Roberta Iacona ${ }^{\$ 1}$, Kate Cross $^{1}$, Simon Blackburn ${ }^{1}$, Edward Kiely ${ }^{1}$, Simon \\ Eaton $^{2}$, Joe Curry ${ }^{1}$, Paolo De Coppi ${ }^{1,2}$ \\ ${ }^{\$}$ Equal contributing first authors \\ ${ }^{1}$ Specialist Neonatal and Paediatric Surgery, Great Ormond Street Hospital, London WC1N 3JH, UK. \\ ${ }^{2}$ Stem Cells and Regenerative Medicine Section, DBC, Institute of Child Health, University College \\ London, London WC1N 1EH, UK.
}

Corresponding author:

Paolo De Coppi, MD, PhD

NIHR Professor of Paediatric Surgery

Nuffield Professor of Paediatric Surgery

Head of Stem Cells \& Regenerative Medicine Section

Developmental Biology\& Cancer Programme

Consultant Paediatric Surgeon

Great Ormond Street Hospital

Surgery Offices

UCL Institute of Child Health

30 Guilford Street

London WC1N 1EH 


\section{Abstract}

Aim of the study: Divided colostomy (DC) has been recommended in anorectal malformations (ARMs) with pre $\neg$ viously reported advantages of decreasing overflow into the distal limb and urinary tract infections (UTIs). Skin bridge loop colostomy (LC) is a technically easier alternative without an increase in these complications. We report our institutional experience of LC in ARM.

Methods: Retrospective study (Institution-approved Clinical Audit) reviewing the clinical records of all patients with ARM undergoing stoma formation in a single UK tertiary pediatric surgical center (2000-2015). Data collected in רcluded type of ARM, associated anomalies, type and level of colostomy, time to stoma closure, complications and UTIs. Results: One hundred and eighty-two (95 female) patients underwent colostomy formation for ARM. The vast major 7 ity (171/ 94\%) underwent LC; 9 (5\%) had a divided colostomy (DC) and $2(1 \%)$ had no available data. The spectrum of defects in girls included rectovestibular (62/65\%), rectovaginal (4/4\%) and cloaca (29/31\%). In boys, 71 (82\%) had a fistula to the urinary tract and $16(18 \%)$ presented with a perineal fistula. Urological abnormalities coexisted in 87 (47.8\%) patients. Thirty five (21\%) patients developed UTIs. Among the 19 girls who developed UTI, 8 had rectovestibular fistula and 11 had cloaca. Of the 16 boys who developed UTI, 14 had a fistula to the urinary tract and 11 had an independent urological abnormality. The mean time from stoma formation to stoma closure was 10 (3-52) months. Complications were reported in 22 (12\%) LCs. Fifteen patients $(9 \%)$ developed a stoma prolapse following LC with $10(6 \%)$ requiring surgical revision.

Conclusions: This is the largest reported series of outcomes following LC for ARM. LC is easier to perform and to close, requiring minimal surgical access, with comparable complications and outcomes to those published for DC.

Type of study: Retrospective comparative study. 


\section{Introduction}

Colostomy formation is often the first surgical procedure in the staged approach to correction of anorectal malformation (ARM). It allows decompression of bowel while awaiting definitive anorectal reconstructive procedures and it is usually maintained 6 to 8 weeks after the perineal procedure to continue diversion of the fecal stream and minimize the risk of surgical site infection. As most surgical procedures, it is associated with complications and morbidity including stoma prolapse and retraction, parastomal hernias and skin excoriation. The optimal type of colostomy for ARM has long been a topic of discussion in pediatric surgery.

colostomy has increased in popularity $[1,2]$. DC is proposed to aid com $\neg$ plete diversion of fecal matter and hence potentially reduce the risk of UTIs. However, DC has the disadvantage of being more invasive at both the initial procedure and stoma closure with the potential for intraabdominal adhesion formation. Stoma bag application can also be problematic in small infants when the stoma is divided. An interesting meta-analysis recently published showed that the 2 procedures are overall comparable, with the only difference being related to the higher incidence of prolapse of LC [3]. In accordance to that study, we have observed, in a large cohort of patients who underwent colostomy in a single institution, that LCs for ARM are safe to perform and have comparable outcome to the ones published for DC.

\section{Methods}

Following approval and registration as a clinical audit, case records of all patients who underwent colostomy formation for anorectal malformation were reviewed retrospectively for the 15 year period between January 2000 and January 2015 at Great Ormond Street Hospital for Children. Two patients were excluded because operative notes did not mention whether the stoma was LC or DC. All patients with cloacal extrophy and conjoint twins were also excluded from the study. Case notes, operative records and clinic letters were examined for demographic data, type of stoma (LC and DC), level of colostomy (sigmoid, transverse), time to stoma closure (months), urinary tract infections (UTIs) and surgical complications including stoma prolapse, stoma retraction, needing for revision, parastomal hernia, bowel obstruction and skin excoriation. Associated urological and spinal anomalies associated were also considered. UTI was defined as 
urinary symptoms associated with a positive culture and treated with antibi-otics. Statistical analyses were conducted using Fisher's Exact Test.

Our typical stoma for ARM is placed at proximal sigmoid level imme 2 diately distal to the descending colon which has a normal retroperito $\neg$ neal attachment and we use a skin bridge. Transverse colostomies were always used for cloaca. 


\section{Results}

One hundred and eighty-two (95 female) patients underwent colos-tomy formation for ARM. Of these, 171 (94\%) underwent LC, 9 patients (5\%) had a divided colostomy (DC) and 2 (1\%) had no available data and were therefore excluded for further analysis. The spectrum of defects in girls included rectovestibular (62/65\%), rectovaginal (4/4\%) and cloaca (29/31\%). In boys, 71 $(82 \%)$ had a fistula to the urinary tract and $16(18 \%)$ presented with a perineal fistula. Urological abnormalities coexisted in $87(47.8 \%)$ patients and included vesicoureteric reflux, duplex systems, ectopic kidney, dysplastic kidney, cystic kidney, fused kidneys, urethral duplication and urethral stenosis. Nineteen children $(11 \%)$ had spinal anomalies including caudal regression, spinal dysraphism, spinal lipoma, tethered cord and spina bifida.

Of the 171 infants who underwent LC, 132 (77\%) were sigmoid colostomies and $39(23 \%)$ transverse colostomies. Transverse colostomy was always performed on cloaca (29) and on surgeon's preference. Seventy nine (46\%) patients were on prophylactic antibiotics. A total of 35 (21\%) patients developed UTIs of which $32(91 \%)$ were already on prophylactic antibiotics.

Fourteen children in this group had documented VUR and 9 patients had a neurogenic bladder. Among the 19 girls who developed UTI, 8 had rectovestibular fistula and 11 had cloaca. Of the 16 boys who developed UTI, 10 had a fistula to the urinary tract (4 bladder neck, 4 bulbar urethra and 1 prostatic urethra). Of the 9 children who had DC, 4 (44\%) developed UTIs. The rates of UTI in LC and DC showed no statistically significant difference (36/171 vs 4/9; p = 0.104). (See Table 1.)

\begin{tabular}{|c|c|c|}
\hline Demographics & LC (171) & DC (9) \\
\hline Female & $89(52 \%)$ & $5(56 \%)$ \\
\hline Low ARM & $73(43 \%)$ & $3(33 \%)$ \\
\hline High ARM & $74(43 \%)$ & $2(22 \%)$ \\
\hline Cloaca & $24(14 \%)$ & $4(44 \%)$ \\
\hline Urological anomalies & $87(47.8 \%)$ & $6(67 \%)$ \\
\hline
\end{tabular}

The duration of stoma (from formation to closure) varied from 2 to 52 months (median 8 months, distribution summarized in Fig. 1). 


\section{Histogram of Stoma duration}

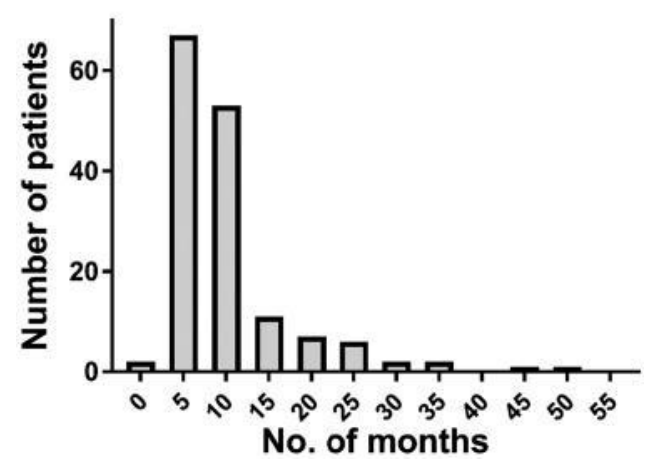

Fig. 1. Duration of stoma in children with LC

The median duration of DC was 9 months. Complications were reported in $22(12 \%)$ children with LC and $4(44 \%)$ with DC $(\mathrm{p}=0.026)$. Fifteen patients $(9 \%)$ developed a stoma prolapse and 7 patients had skin excoriation following LC. The complications categorized by duration of stoma are summarized in Fig. 2.

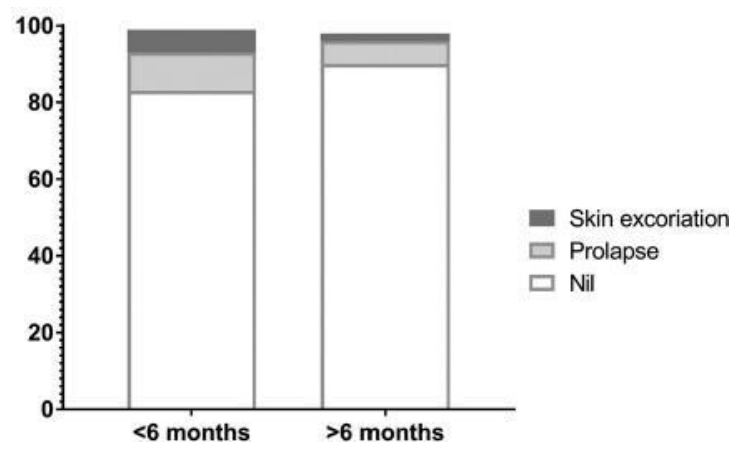

Fig. 2. Percentage of each complication in LC in the children who had stoma closure within 6 months or after 6 months.

We analyzed for correlation between prolapse and duration of stoma ( $<6$ months vs $>6$ months).

In the LC group, 7 patients continue to have a stoma in situ and 8 patients did not have date of closure in the notes. Among the remaining $163 \mathrm{LCs}$, there was no difference in prolapse rates between those who had stoma closure within 6 months $(\mathrm{p}=0.40,2$ tailed Fisher's exact test).

The prolapse rate was significantly higher in transverse colostomies $(8 / 39$ vs 7/132, p = 0.027, Fig. 3). 


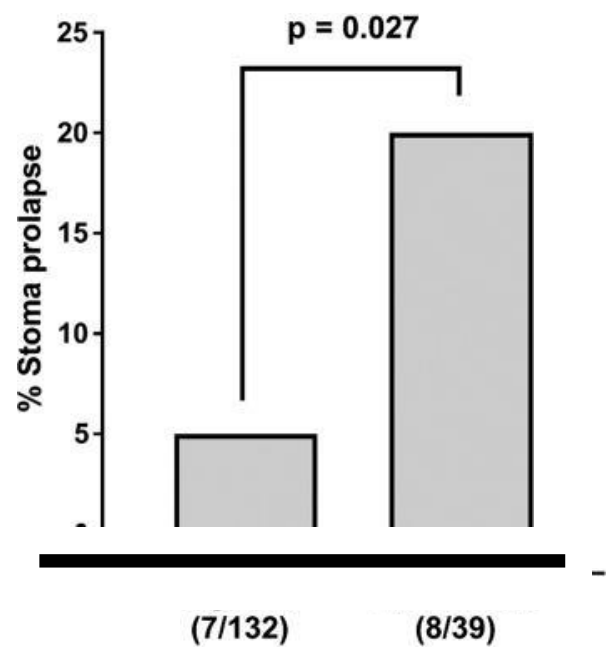

Fig. 3. Comparison of stoma prolapse rates between sigmoid and transverse LC.

Ten of the prolapsed loop stomas needed surgical revision. There was no significant difference in the rate of stoma revisions between transverse LC and sigmoid LC (5/ 132 vs 4/39, p = 0.239, Fig. 4).

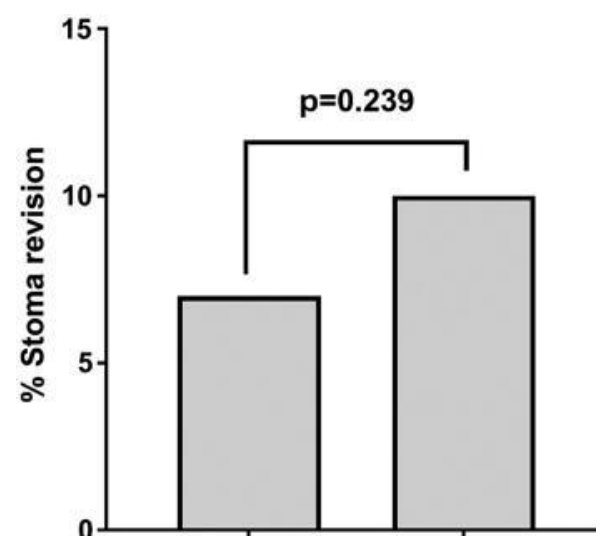

Fig. 4. Comparison of stoma revision rates for prolapse between sigmoid and transverse LC 
Seven children with LC had skin excoriation. Among the 9 children with DC, 3 needed revision of stoma ( 2 stoma prolapses and one inade $\neg q u a t e ~ l e n g t h$ for definitive procedure). There was no significant differาence in the rate of stoma prolapse between LC and DC (15/171 vs $2 / 9, \mathrm{p}=$ 0.204, figure 6). One infant had skin excoriation. 


\section{Discussion}

Colostomy is performed in staged surgery for ARM to relieve the intestinal obstruction and allow bowel decompression prior to definitive reconstruction. However, the ideal type of colostomy has been debated by pediatric surgeons [3-8]. A divided colostomy (DC) created at the junction of the descending and sigmoid colon has been recommended [2]. Our practice, however, has been to perform loop colostomy with the ease of surgical technique both at performing and closing the stoma and managing of the stoma bag. Our anecdotal experience had been one of no differences in the incidence of UTI between LC and DC. This 15 year retrospective data presented here reinforce our preference and indicate that LC is safe in patients affected by ARM.

Divided colostomy was recommended by Pena with the proposed advantages of diverting fecal flow to the distal pouch, avoiding fecal impaction and reducing the risk of UTI [1]. The reported complications include excoriation, prolapse, stenosis/ obstruction, bleeding, parastomal herniae, skin excoriation and skin dehiscence $[9,10]$. However our results suggest that the presence of 2 ends of the stoma in the same stoma bag does not increase the risk of complications including UTI and distal bowel dilatation. Interestingly, a meta-analysis recently published by this journal revealed that there are no significant differences in stoma retraction, necrosis, parastomal hernia or peristomal skin breakdown [3]. All these complications are also present in our series with no significant differences to the reported incidence of those in DC. The meta-analysis also indicates that there is also no difference on the rate of UTI.

Regardless of the type of stoma (LC vs DC) the most important criterion for successful anorectal reconstruction is the availability of ad-equate distal bowel to use for the definitive surgery. We therefore use transverse colon for cloacal malformations and proximal sigmoid colon for other types of ARM.

It has been proposed that the disadvantage for LC has been the risk of UTIs, especially in those patients who have a fistula between the rectum and urinary tract [1]. Indeed, the rate of UTIs has previously been reported to be significantly higher following colostomy formation in ARM with VUR, renal dysplasia and neurogenic bladder [11]. However, it is now clear that high rates of UTI are associated with ARM indepen $\neg$ dent of the type of colostomy [8]. Sanchez et al. recently reported rates of 39\% UTI in children with ARM and associated VUR even in the presence of continuous antibiotic prophylaxis and with a DC [12]. Sim-ilarly, Liechty et al. showed that patients receiving an LC are not at higher risk of developing UTI compared to the one receiving a DC [16]. In the light of this new evidence it appears that children with ARM are more susceptible to UTIs independently from the type of stoma they received, and the only risk factor is the association of anomalies of the genitourinar genitourinary system $[8,16]$. Our study supports the finding by demon $\neg$ strating a comparable rate of UTI on LC to the small series of DC included here and most importantly to previously published rates for DC [11]. 
Stoma prolapse has been one of the significant complications reported for colostomies. A few studies have reported increased rates of prolapse following LC compared to DC [7-9]. However, colostomies in those reports were predominantly located at the transverse colon, which we also observed to be associated with the majority of prolapses. This is in keeping with the idea that stoma formed in the more mobile parts of the colon may be more likely to prolapse. Transverse colostomies are safe in cloacal anomalies as they ensure the preserva $\neg$ tion of adequate distal bowel for later reconstruction, especially as some patients may also require vaginal replacement using bowel to achieve a functional genital tract. In ARM we generally prefer to place the stoma at a very proximal sigmoid level immediately distal to the descending colon which has a normal retroperitoneal attachment to circumvent this potential problem. Moreover, the loop colostomies were performed using a skin bridge, which is believed to reduce the risk of prolapse similarly to the use of subcutaneous tunnel for bowel exit, purse string sutures at fascial level, anchoring U stitch and using a rubber catheter under the mesenteric border of the loop in the initial postoperative period described [13-17]. Similar to the series from Demirogullari and van den Hondel, the prolapse rate in our series is comparable to those published following divided colostomies $[8,11,18]$. The risk of prolapses was reported previously to be higher when a long period between formation and closure of loop colostomies [5]. However, this seems not always be related to the type of stoma since Almossalam et al. published a series of 55 LCs and 49 DCs and did not find any difference between complications in the 2 groups regardless of the duration of stoma [4]. Our data have similarly demonstrated no difference in prolapse rates in those who had stoma closure within or after 6 months.

Another argument for DC has been that complete fecal diversion may reduce the risk of distal bowel dilatation and therefore of resultant long term incontinence. We have not analyzed the long term functional outcomes in this cohort. However we have not had to perform distal bowel tapering procedures at the definitive reconstruction. We perform a thorough distal bowel washout during the initial stoma formation and believe that this minimizes the occurrence of persistent distal bowel dilatation.

Despite the limitation of being a retrospective study with a very limited number of control DCs, this represents the largest reported series of stoma related outcomes following LC for ARM. LC is easier to perform and to close, and has comparable complications and outcomes to those published for DC. 


\section{References}

[1] Pena A, Migotto-Krieger M, Levitt MA. Colostomy in anorectal malformations: a procedure with serious but preventable complications. J Pediatr Surg 2006;41:748-56.

[2] Pena A, Devries PA. Posterior sagittal anorectoplasty: important technical considerations and new applications. J Pediatr Surg 1982;17:796-811.

[3] Youssef F, Arbash G, Puligandla PS, et al. Loop versus divided colostomy for the management of anorectal malformations: a systematic review and meta-analysis. J Pediatr Surg 2017;52:783-90.

[4] Almosallam OI, Aseeri A, Shanafey SA. Outcome of loop versus divided colostomy in the management of anorectal malformations. Ann Saudi Med 2016;36:352-5.

[5] Gardikis S, Antypas S, Mamoulakis C, et al. Colostomy type in anorectal malformations: 10-years experience. Minerva Pediatr 2004;56:425-9.

[6] Kroese LF, de Smet GH, Jeekel J, et al. Systematic review and meta-analysis of extraperitoneal versus transperitoneal colostomy for preventing parastomal hernia. Dis Colon Rectum 2016;59:688-95. [7] Oda O, Davies D, Colapinto K, et al. Loop versus divided colostomy for the manage-ment of anorectal malformations. J Pediatr Surg 2014;49:87-90. 